FABIAN WINTER

\title{
PHARMAKON UND FORMATION: ABY WARBURGS ORDNUNGSFORMATE DER PSYCHE
}

\section{Embdens Diagnose}

\begin{abstract}
Doch hat der Arzt dabei oft den Eindruck, daß Patient vor der eigentlichen wissenschaftlichen Produktion in das Bücherkaufen flüchtet, wie denn auch immer neue Gestaltungen seines Arbeitsmechanismus, Schreibtisch, Federn, Bleistifte, Zettelkästen, Lesepulte, eine ganz unverhältnismäßig große Rolle in seinem Tun und Reden spielen. ${ }^{1}$
\end{abstract}

1 Heinrich Embden: Anamnese Warburg, in: Ludwig Binswanger, Aby Moritz Warburg: Die unendliche Heilung: Aby Warburgs Krankengeschichte, hg. v. Chantal Marazia, Davide Stimilli, Zürich 2007 , 260-262, hier 261.

2 Im Anschluss an Jacques Derridas Lektüre von Platos Phaidros: «Pharmakeia ist auch ein allgemeiner Name, der die Verwaltung des pharmakon, der Droge bedeutet: des Heilmittels und/oder des Giftes." Jacques Derrida: Dissemination, Wien $1995,78$.

3 Der Symptom-Begriff kann hier nur so weit erläutert werden, als dass solche Symptome in der modernen Psychotherapie entweder als dysfunktionale oder funktionale Selbstheilungsversuche betrachtet werden. Ob Warburgs Symptome derart eingeordnet werden können, hinge davon ab, ob die Lösungsversuche der Ordnungstechniken insgesamt als produktiv für die eigentlichen Vorhaben gewertet werden. Wenn Warburg also Stifte und Zettel sortieren muss, um dann sehr produktiv arbeiten zu können, wären seine Symptome als funktional tolerierbar. Es könnte daher auch die These aufgestellt werden, dass Warburgs Symptome zwischen 1918 und 1924 dysfunktional werden. Für produktive Hinweise aus Sicht aktueller psychotherapeutischer Praxis danke ich Mirja Seidler. 4 Embden: Anamnese Warburg, 261. 
5 Vgl. Markus Krajewski: ZettelWirtschaft. Die Geburt der Kartei aus dem Geiste der Bibliothek, Berlin 2017, $147 \mathrm{f}$.

6 Vgl. Davide Stimilli: Tinctura Warburgii, in: Binswanger, Warburg: Die unendliche Heilung, 7-26. Vgl. Michael Diers: Kreuzlinger Passion, in: kritische berichte - Zeitschrift für Kunst- und Kulturwissenschaften, Bd. 7, Nr. 4/5, 1979, 5-14.

7 Binswanger, Warburg: Die unendliche Heilung, 219.

8 Ebd., 220.

9 Ebd.

10 Heinrich Embden: Brief an Hans Berger (23.10.1920), in: Binswanger, Warburg: Die unendliche Heilung, 255-257, hier $255 \mathrm{f}$.

11 Ebd., 257.

12 Ebd. dass diese Ordnungstechniken vielmehr notwendige Bedingung der Konstitution von Warburgs Existenz als Gelehrter sind. Wenn hier nach einer Agentialität der gebildeten Formate als Psychotechniken ${ }^{5}$ gefragt wird, dann nicht mit dem Ziele einer erneuten Nosographie Warburgs, sondern um Zugänge einer Medientheorie des Formate-Schaffens zu erproben. Dafür werden insbesondere die pathologisierten Ordnungstechniken Warburgs in den Blick genommen und als Ausgangspunkt einer Wirkmacht betrachtet, die sowohl für den Prozess der Genesung als auch für die kulturwissenschaftliche Forschung des Patienten konstitutiv ist und durch das pharmazeutische Medium des Schreibens konstitutiert wird. Das ärztliche Missverstehen der Bedeutung von Organisationstechniken für den Gelehrten als Substanz der ärztlichen Psychopathologie ist dafür der Ausgangspunkt. Beginnend bei den medizinischen Formularen und Berichten kann Warburgs Krankengeschichte parallel zu seinem eigenen Formate-Schaffen erzählt werden, welches in den Diagnosen erst als Droge und Gift, später dann als Heilmittel und Rettung beschrieben wird. ${ }^{6}$

Zunächst ist es keinesfalls ein Missverständnis, dass Warburg im November I9 8 infolge eines Selbstmordversuchs in der Hamburger Klinik von Arnold Lienau aufgenommen und bis Juli r9ı9 behandelt wird. Ende Mai I9I9 notiert Lienau, dass Warburg «um Pistole [bittet], da er sich mit Referent erschießen wolle. Halluzinationen zeitweise sehr lebhaft». ${ }^{7}$ Trotzdem wird Warburg Anfang Juli versuchsweise «[u]ngeheilt, aber etwas gebessert entlassen», ${ }^{8}$ da er zusagt, sich wieder in stationäre Behandlung zu begeben, «wenn das <Experiment> nicht glückt». ${ }^{9}$ Warburg wird im Oktober 1920 wieder als Patient aufgenommen und verbringt sieben Monate in einer Jenaer Klinik unter Leitung von Hans Berger. Embden beschreibt Berger in einem Brief die Diagnose ihres gemeinsamen Patienten als äußerst diffizil: «Bei dem enorm bunten Krankheitsbild, aus dem ich heute nur die Beeinträchtigungsideen herausgegriffen habe, ist ja eine Diagnose sehr schwierig. Die Hauptsache ist doch jedenfalls die angeborene Psychopathie mit ihrer Neigung zu Zwangsvorstellungen und Handlungen». ${ }^{10}$ Was hier als eine angeborene Krankheit beschrieben wird, für die die Zwangshandlungen mehr Symptome als Ursachen zu sein scheinen, wird mit Blick auf die intensiv angewandten Organisationstechniken Warburgs jedoch im nächsten Satz als Teil der Krankheitsursache vermutet: Der Arzt hat «oft den Eindruck, daß die Verdächtigungen mehr sekundär, nur Stütze und Rechtfertigung seiner durch Zwang bedingten Lebensweise herangezogen und geglaubt würden. Feindlich ist alles, was seinen Phobien und der schrankenlosen Betätigung seiner pathologischen Zeremonien störend und einschränkend in den Weg tritt»." Embden gibt in diesem Brief vom 23. Oktober I920 auch ein konkretes Beispiel einer von ihm pathologisierten Schreibtechnik: «Das Tagebuchschreiben ist absoluter Zwang, er übt es ohne jede Rücksicht auf Besucher [aus]». ${ }^{12}$ Nach sieben Monaten in Jena überweist Berger Warburg in das Sanatorium von Ludwig Binswanger in Kreuzlingen. Für Warburgs Aufnahme in der Schweizer Klinik verfasst Embden besagten Anamnesebericht, der das von 
ihm identifizierte Zusammenwirken von Krankheit und Organisationstechniken hervorhebt. Außerdem verbindet Embden in seinem Bericht zu Warburgs Verfassung zeithistorische Ereignisse mit beruflichen Faktoren:

Für seine vornehmliche wissenschaftliche Aufgabe: Weiterleben antiker Gedanken im <Mittelalter $>$ hatte er sich der Erforschung der Astrologie pp gewidmet. Hierbei glitt er allmählich aus dem historischen Standpunkt in den halb gläubigen, resp. abergläubischen Mimiker. - Vor der Katastrophe, die von der des Vaterlandes aus gelöst [sic] wurde, war er soweit gekommen, daß er sich für einen Werwolf hielt. Er glaubte, drohendem Unheil nur durch Tötung seiner Familie und Selbstmord entgehen zu können, ergriff einen Revolver, wurde leicht entwaffnet und Anfang Nov. I9 8 in die Klinik von Dr. Lienau überführt. Während des Krieges waren seine Briefe zunehmend spärlicher (an Inhalt), schlechter geschrieben und flüchtiger geworden. ${ }^{\mathbf{1 3}}$

Erstens wird ein Abgleiten Warburgs im Rahmen seiner kulturwissenschaftlichen Forschung diagnostiziert, das sich vom klaren Vorhaben - dem Nachweis des Nachlebens der Antike - zu einem abergläubischen Unternehmen bewege. Zweitens habe der Weltkrieg als «Katastrophe [...] des Vaterlandes ${ }^{14}$ jene persönliche Katastrophe Warburgs initialisiert, vor der er sich und seine Familie durch Selbstmord zu retten hoffte. Drittens hätten Warburgs Briefe an Qualität und Quantität seit dem Weltkrieg abgenommen, und eine Qualitätsbeurteilung der Briefe ist es dann auch, die 1924 zwei Monate vor seiner Rückkehr nach Hamburg als Indiz der Genesung Warburgs dient: «Schreibt seit seiner Krankheit jetzt zum ersten Mal wieder mit Tinte, was ihn eine große Überwindung gekostet hat, und viel ausführlicher». ${ }^{15}$ Der Erste Weltkrieg mag aus Embdens Sicht Anlass für Warburgs Krankheitsausbruch gewesen sein. Für die Argumentation des vorliegenden Beitrags ist das jedoch weniger produktiv als der Umstand, dass die Qualität von Warburgs kulturwissenschaftlicher Forschung sowie die der damit verbundenen Formations- und Kulturtechniken des Organisierens, Anordnens und Schreibens stark variierte. Zunächst sollen daher die von Warburg genutzten Formate und Formationen genauer betrachtet werden, bevor ihre Rückwirkung als Pharmazeutika der wissenschaftlichen Praktiken Warburgs fokussiert wird.

\section{Formate als Pharmazeutika}

Als Pharmakon kann nicht nur das Briefeschreiben, sondern auch die Arbeit der Überführung der Briefe in ein neues Format identifiziert werden, welche zum Bestandteil der Psychodiagnose des Patienten Warburg gemacht wird. Dabei handelt es sich um eine Organisationstechnik, die Warburg von I905 bis I 9 I 8 nutzt und die heute weitestgehend vergessen ist: das sogenannte Briefkopierbuch. Michael Diers hat bereits i99 I auf die besondere Quellenlage der erhaltenen Korrespondenz Warburgs aufmerksam gemacht und mit seiner Studie Warburg aus Briefen auf die besondere Archivierung versandter Briefe in Briefkopierbüchern hingewiesen. ${ }^{16}$ Vor der Etablierung der Schreibmaschine,
13 Embden: Anamnese Warburg, 262. 14 Ebd.

15 Ludwig Binswanger, Kurt Binswanger: Krankengeschichte Kreuzlingen 1921-1924, in: Binswanger, Warburg: Die unendliche Heilung, 33-92, hier 9o.

16 Vgl. Michael Diers: Warburg aus Briefen. Kommentare zu den Kopierbüchern der Jahre 1905-1918, Weinheim 1991. 
17 Vgl. Delphine Gardey: Schreiben, Rechnen, Ablegen. Wie eine Revolution des Bürolebens unsere Gesellschaft verändert hat, Göttingen 2019, 129.

$18 \mathrm{Vgl}$. Cornelia Vismann: Akten. Medientechnik und Recht, Frankfurt/M. 2011, 277.

19 Zur Mediengeschichte des Briefkopierbuches forscht der Autor im Rahmen seiner Dissertationsschrift. Eine der wenigen kulturwissenschaftlichen Betrachtungen von Briefkopierbüchern um 1900 findet sich in: Konrad Heumann: Archivierungsspuren, in: Anne Bohnenkamp, Waltraud Wiethölter (Hg.): Der Brief, Ereignis \& Objekt, Katalog, Frankfurt/M. 2008, 263-315.

20 Vgl. Diers: Warburg aus Briefen, $27 \mathrm{f}$.

21 Derrida: Dissemination, 104.

22 Vgl. Diers: Warburg aus Briefen, 30.

23 Dies kann auch aus Warburgs an die Mutter gerichtetem Wunsch abgeleitet werden, den Hamburger Mittelweg-Schreibtisch 1897 nach Florenz zu überstellen, da Warburg gerne an diesem Möbel arbeiten möchte. Warburgs Mutter lehnt diesen Wunsch aus Kostengründen allerdings ab. Vgl. Thomas Hensel: Warburg's Desk, in: Philosophy of Photography, Bd. 8, Nr. 1, 2017, 53-74, hier 54 .

24 Ebd., 61.

25 Karl Jaspers: Wesen und Kritik der Psychotherapie, München 1955, 12. der Möglichkeit zur zeitgleichen Herstellung einer Durchschlagkopie ${ }^{17}$ und dem damit korrespondierenden Ablage- und Archivierungssystem des Stehordners ${ }^{18}$ musste geschäftlich versandte Korrespondenz durch Abschrift oder jene Schwellentechniken des Briefkopierens erfolgen, zu der auch das von Warburg genutzte Verfahren der Nasskopie gehört. ${ }^{19}$ Kennengelernt hat Warburg das Verfahren vermutlich im kaufmännischen Betrieb seiner Eltern, ${ }^{20}$ was zum historischen Milieu dieser Kopiertechnik führt. Wie Jacques Derrida in seiner Analyse des Phaidros-Dialogs herausstellt, teilt die rationale Lebenswelt der merkantilen Schreibstube denselben Gott wie jene Welt des Aberglaubens, in die Warburg nach Embden am Ende seiner Tätigkeit als BriefkopierbuchFührer abgleitet:

[Der] Gott des Rechnens, der Arithmetik und der rationalen Wissenschaften befiehlt auch über die okkulten Wissenschaften, die Astrologie, die Alchimie. Er ist der Gott der magischen Formeln, die das Meer besänftigen, der geheimen Erzählungen, der verborgenen Texte: der Archetyp des Hermes, des Gottes des Kryptogramms nicht weniger als der Graphie. ${ }^{21}$

Warburg der Briefeschreiber, das ist bis I9 18 auch Warburg der Briefkopist. Ab seinem Nervenzusammenbruch ${ }^{22}$ nutzt Warburg zum Kopieren der von ihm versandten Briefe zumeist die Kombination von Schreibmaschine und Kohledurchschlagpapier. Wie medienhistorisch die Bedeutung der Kulturtechnik des Briefkopierbuch-Führens sowie das Wissen um dieselbe verebbt, so wird auch Warburg nach seiner Psychose I9 8 kein Kopierbuch seiner Briefe mehr führen. Dies bedeutet keinesfalls, dass der in den Kliniken forcierte Milieuwechsel des Patienten und die Psychopathologisierung der Ordnungstechniken Warburgs nach I9I 8 oder 1924 eine Abstinenz von Formaten bedeutet. Thomas Hensel hat nachdrücklich die Bedeutung des Schreibtisches als Organisationseinheit des Wissens für Warburg herausgestellt, der als Habitat und Zentrum intellektueller Arbeit eine Konstante für Warburg verkörpert. ${ }^{23}$ Der Schreibtisch als bevorzugtes Aktionszentrum werde auch in Kreuzlingen gleichsam zum Anker und zur Arznei: «Towards the end of his stay in Kreuzlingen [...] Warburg's desk becomes his anchor and a remedy and changes to it or on it spark crises and compulsive responses». ${ }^{24}$

Karl Jaspers schreibt zur Kritik der Psychotherapie um I900, dass die Heilung psychisch Kranker «durch Modifikation der Lebenssituation» angestrebt werde, deren allgemeinstes Verfahren der sogenannte «Milieuwechsel» sei, ${ }^{25}$ wozu offensichtlich auch eine extrinsische Unterbrechung aller bisher genutzten Ordnungstechniken Warburgs gehört. Für eine kurze Zeitspanne wird Warburg einer solchen Milieu-Intervention ausgesetzt, die sich aber auf die Gesundung des Patienten kaum auswirkt. Vielmehr lässt sich feststellen, dass die Palette an Wissensformationen aus mittelfristig wechselnden Techniken (Briefkopierbuch und Bibliothekstagebuch) und langfristigeren Organisationstechniken (Zettelkasten und Bibliothek) vor, während und nach der Krankheitsphase besteht und 
in Kreuzlingen einzelne Organisationstechniken sogar von ihm intensiver betrieben werden, auch wenn etwa das Führen eines Briefkopierbuches mit dem ersten Milieuwechsel Warburgs für immer beendet sein wird.

Andere Organisationstechniken werden aber gerade in Kreuzlingen von Warburg in deutlich intensivierter Form genutzt, insbesondere jene, die im direkten Zusammenhang mit seinem zunehmend primären Unternehmen einer Formation seines Wissens stehen, der Kulturwissenschaftlichen Bibliothek Warburg (K.B.W.). Seit I904 arbeitet er energisch an der Öffnung und Institutionalisierung seiner Bibliothek, die schon vor I9I 8 einen bemerkenswerten Ruf als halböffentliche Forschungseinrichtung hat. ${ }^{26}$ Während seiner Zeit in psychiatrischer Behandlung organisiert Warburg den sukzessiven Ausbau durch den Ankauf der Bestände aus der Bibliothek Franz Bolls sowie den Neubau, in den die K. B.W. I926 einzieht. Aus Kreuzlingen schreibt Warburg am 27. Juli I924 an seinen Bruder Paul, dass «die Basis verbreitert werden [muss]», ${ }^{27}$ da die Bibliothek als Warburg-Seismograph nur durch gezielte Ankäufe und räumliche Expansion ihren Dienst erfüllen kann: «[I] ch muß Räume haben, nach links: Vortragssaal[,] in der Mitte: Bibliothek, und rechts: Wohnhaus [...]. Erst auf dieser um 2/3 erweiterten Basis kann der Seismograph funken; nichts ist [...] dafür zu hoch bezahlt». ${ }^{\mathbf{2}}$ Aby Warburg, der die größten Teile seiner Forschungen über das Vermögen seiner Brüder finanzierte, verspricht in diesem Brief seinem Bruder Paul Warburg, dass sich die Kosten für das Unternehmen der K. B.W. als das Denken organisierende Institution lohnen werden. Die Bibliothek wird von Aby Warburg als Denkorgan und Akteur der eigenen Forschung verstanden und soll von Paul Warburg ebenfalls als Teil des eigenen Denkapparates erkannt werden: $\ll[\mathrm{I}] \mathrm{ch}$ werde nicht müde werden in dem Versuch, den geistigen Menschen bei Dir herauszuholen, ein Geschäft, das ich bei Felix [Warburg, Bruder von Aby und Paul Warburg, FW] leider aufgegeben habe: ich will keine eilig die Welt durchjagenden Menschen, die bei Ankunft schon nach der Abfahrt jappen, wenn ich ihnen klarmachen soll, welche Bedeutung ihr eigenes Denkorgan, die Bibliothek Warburg, zu erringen beginnt». ${ }^{29}$ Die Bedeutung der eigenen Bibliothek als Denkorgan für Warburg, seine Brüder wie auch jede_n Besucher_in wird im Anamnesebericht ebenfalls erkannt, jedoch völlig anders ausgelegt.

[Warburg k]ennt keine Trennung zwischen Arbeits- und Mußestunden. Dauernd in Bibliothek und am Schreibtisch. Sammelt ein ungeheures, sehr wohlgeordnetes Material in seinen Zettelkästen. Vor jedem Fertigmachen große Angst. Terminangst höchsten Grades. Jeder Vortrag verschoben. Die sehr spärlichen Publikationen immer verspätet. Korrekturlesen einer kleinen Abhandlung als furchtbare Veranstaltung behandelt. Patient dann wochenlang krank. Sehr komplizierte, äußerlich pedantische Veranstaltungen dabei: Ringbücher, bunte Stifte, Abschriften pp. ${ }^{30}$

Warburgs Palette an Formationstechniken artikuliert sich nicht in einer Vielzahl von Publikationen, sondern im Schaffen und Prozessieren von Ordnungsund Schreibtechniken, wie dem Zettelkasten, dem Briefkopierbuch oder dem
26 Vgl. Fritz Saxl: Die Geschichte der Bibliothek Warburg, übers. v. Matthias Fienbork, in: Ernst $\mathrm{H}$. Gombrich: Aby Warburg. Eine intellektuelle Biographie, Hamburg 2012, 433-450, hier $433 \mathrm{f}$.

27 Binswanger, Warburg: Die unendliche Heilung, 117.

28 Ebd.

29 Ebd., 116.

30 Embden: Anamnese Warburg, 261. 
31 Ebd.

32 Saxl: Die Geschichte der Bibliothek Warburg, $43^{8}$.

33 Vgl. Markus Krajewski: Mobility on slips. Or: How to invest in paper. The Aby Warburg style, in: Philosophy of Photography, Bd. 8, Nr. 1/2, 2017, 97-108.

34 Daniel Schatz: Das Bibliothekskonzept Aby M. Warburgs im Vergleich mit modernen Methoden der Sacherschließung, Fachhochschule Potsdam 2002, docplayer.org/15671928-Dasbibliothekskonzept-aby-m-warburgs-imvergleich-mit-modernen-methoden-dersacherschliessung.html (16.6.2019). 35 Vgl. Björn Biester: Tagebuch der Kulturwissenschaftlichen Bibliothek Warburg, 1926-1929. Annotiertes Sach-, Begriffs- und Ortsregister, Erlangen 2005.

36 Embden: Anamnese Warburg, 261.

37 Binswanger, Warburg: Die unendliche Heilung, $116 \mathrm{f}$.
Bibliothekstagebuch, welche Embdens Bericht nach «eine ganz unverhältnismäßig große Rolle in seinem Tun und Reden spielen». ${ }^{31}$ Auch Fritz Saxl beschreibt die Intensität, mit der Warburg seine Formate, insbesondere seinen Zettelkasten führte, als Ausdruck von Warburgs «Gelehrtenexistenz». ${ }^{32}$ Aus unterschiedlicher Perspektive beschreiben Saxl und Embden, wie Warburg dazu prädestiniert war, als Privatgelehrter zu existieren. Dies bedeute weniger, große Theorien zu schaffen, sondern vielmehr jene Formate zu orchestrieren, die seine Existenz als Gelehrter umgeben: Briefkopierbuch, Zettelkasten, ${ }^{33}$ Aufstellungssystematik ${ }^{34}$ oder Bibliothekstagebuch ${ }^{35}$ - alle Organisationstechniken des Wissenschaftlers kulminieren in der sie umgebenden Formation der K. B.W. Embden sieht in der Zusammenlegung von Wohn- und Arbeitsraum jedoch vor allem einen weiteren Diagnose-Anlass:

Er entwickelte sich, bei seinem Beruf als Privatgelehrter dauernd zu Haus beschäf-
tigt, überhaupt zu einem krassen Haustyrannen und Topfgucker. Bei kleinen Anlässen
hemmungslose Zornausbrüche, er redet dann Dolche, begründet das Maß der Erre-
gung mit glänzender Dialektik so, daß er jede winzige Tatsache sub speciem aeterni
rückt, gerade in dieser Tatsache den Urgrund aller Lebensschwierigkeiten aufzeigt.

\section{Das Pharmakon als Medium des Wissens}

Sub speciem aeterni - unter dem Gesichtspunkt der Ewigkeit wird laut Embden jeder Anlass zur Erzürnung von Warburg derart als «Urgrund aller Lebensschwierigkeiten» überhöht, dass ihm auch die eigene psychische Erkrankung als Wegmarke im Weltgeschehen erscheinen muss. In einem Brief an seinen Bruder Paul beschreibt er die von ihm erkannt geglaubte Verbindung seiner Krankheit mit den Leidensstationen der Menschheit. Diese Erkenntnis lasse sich aber, wie Warburg an seinen Bruder Paul schreibt, nicht innerhalb eines Briefes erklären. Sie könne aber nach der Rückkehr aus Kreuzlingen in die K.B.W. und durch diese erfolgen:

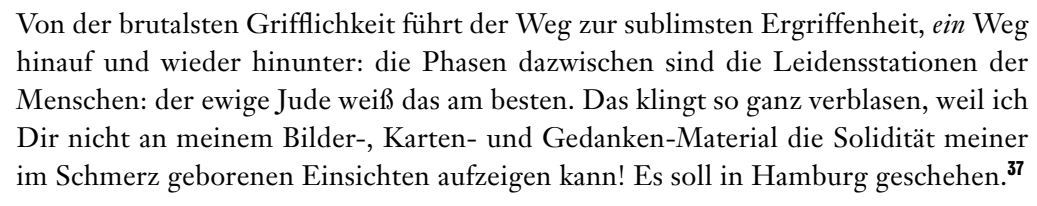

Die Beschreibung des Leidens zwischen «Grifflichkeit» und «Ergriffenheit» wird von Warburg gegen Ende seiner Krankenjahre zu Papier gebracht. Die hier als gemeinsam wirkend erkannten Kräfte lassen bereits die nach Kreuzlingen begonnene Arbeitsmethode am Mnemosyne-Bildatlas als eine des sinnlichen Arrangierens antizipieren. Das Arrangieren aus der Ergriffenheit heraus bildet eine Methode, die gängige Ordnungsformate der Wissenschaft unterläuft und verborgene Ordnungen zwischen den Elementen (kontemplativ) wahrnehmbar macht. Insbesondere Georges Didi-Hubermans Blick auf die 
Montagearbeit in Warburgs Bilderatlas ist in Bezug auf epistemische Dimensionen des Empathischen erhellend:

Er [der Bilderatlas] zerschlägt die selbstproklamierten Gewissheiten sowohl der Wissenschaft, die sich ihrer Wahrheiten, als auch der Kunst, die sich ihrer Kriterien sicher ist. Er erfindet zwischen all dem liegende Bereiche forschenden Erkundens, heuristische Intervalle. Von definitiven Axiomen will er nichts wissen. Denn er beruht auf einer Erkenntnistheorie, die sich dem Risiko des Sinnlichen, und auf einer Ästhetik, die sich dem Risiko der Disparität aussetzt. ${ }^{38}$

Der Zusammenhang zwischen den Ordnungsformaten in wissenschaftlichen Schreibprozessen und der Ordnungsformation des Psychischen werde besonders im phantomhaften Geschichtsverständnis Warburgs deutlich. Im Gegensatz zur Darstellung etwa der Renaissance als Epoche einer produktiven Nachahmung der Antike formuliere Warburg seine Kunstgeschichte als «Phantommodell [...], in dem die Zeit nicht auf die akademische Weitergabe des Wissens projiziert wird, sondern ihren Ausdruck in einer zwanghaften Wiederkehr und einem geisterhaften <Nachleben $>$ der Formen findet. Also im Ungewußten, Ungedachten und Unbewußten der Zeit. ${ }^{39}$ Warburgs Ordnungsformate, die er abseits der etablierten Geschichtswissenschaft sucht und findet, setzen ihn selbst in eine Spannung zwischen Chaos und Ordnung, in deren Zusammenhang seine Psyche zwischen Auflösungen des Selbst und beständigem Herstellen des Selbst durch Zwangshandlungen agiert. Der für Warburg zentrale Begriff der Pathosformel wird für ihn in der ambivalenten Spannung «zwischen dem Wunsch nach Identifizierung und der Notwendigkeit einer Veränderung, zwischen Reinigung und Vermischung, normal und pathologisch, Ordnung und Chaos, Evidentem und Ungedachtem $\gg^{\mathbf{4 0}}$ zum diagnostizierbaren Leiden, dessen Pharmakon Ordnungsformate sind, die Bewegungen zwischen diesen Polen nicht verhindern, sondern erlauben und befördern. Daher wird auch der Schreibtisch, dessen Signifikanz für Warburg als dispositives Element in der Entstehung des Bilderatlas von Hensel unter anderem über die Funktionen des Vermessens und Anpinnens aufgezeigt wird, ${ }^{41}$ bei Didi-Huberman zum Paradebeispiel eines Mobiliars wissenschaftlicher Un/Ordnung: «Die Unordnung ist nur für denjenigen Unvernunft, der sich weigert, die Zerstückelung der Welt zu denken, zu respektieren und in gewisser Weise zu begleiten. Der Tisch [table] wäre also ein vorzüglicher Ort, um diese Zerstückelung (emphatisch) zu versammeln und (abstrakt) zu präsentieren».42

\section{Heilung durch Wissenschaft}

Bevor Ordnungstechniken zu Warburgs dosiertem Pharmakon werden, vergehen knapp sechs Jahre mit psychotherapeutischen Diagnose- und Behandlungsbemühungen, deren allererste Maßnahmen dem entsprechen, was von der noch jungen Fachdisziplin im historischen Kontext erwartet werden kann:
38 Georges Didi-Huberman: Hepatische Empathie: Die Affinität des Inkommensurablen nach Aby Warburg, in: Trivium. Revue franco-allemande de sciences humaines et sociales - Deutsch-französische Zeitschrift für Geistes- und Sozialwissenschaften, Nr. 10, 30.3.2012, 1-19, hier 3.

39 Georges Didi-Huberman: Das Nachleben der Bilder: Kunstgeschichte und Phantomzeit nach Aby Warburg, übers. v. Michael Bischoff, Berlin 2010, $30 \mathrm{f}$.

40 Ebd. 32.

41 Hensel: Warburg's Desk, $69-72$.

42 Didi-Huberman: Hepatische Empathie, $13 \mathrm{f}$. 
43 Karl Jaspers beschreibt die Verfahren der Psychotherapie jener Zeit als «Überrumpelungstherapie» und "aufoktroyierte[n] Milieuwechsel", Jaspers: Wesen und Kritik der

Psychotherapie, 26.

44 Ebd. 27.

$45 \mathrm{Vgl}$. Ludwig Binswanger: Grundformen und Erkenntnis menschlichen Daseins, in: ders.: Ausgewählte Werke in vier Bänden, Bd. 2, hg. v. Max Herzog, Hans-Jürg Braun, Heidelberg 1993.

46 Stimilli: Tinctura Warburgii, 17. 47 Ludwig Binswanger: Formen missglückten Daseins, in: ders.: Ausgewählte Werke in vier Bänden, Bd. 1, 233-418, hier 36o.

48 Binswanger, Warburg:

Die unendliche Heilung, $38 \mathrm{f}$.

49 Ebd., 39.

50 Ebd., 45.

51 Ebd.

52 Stimilli: Tinctura Warburgii, 18.
Medikation und Milieuwechsel..$^{43} \mathrm{Um}$ mehr als Symptome zu lindern, kritisiert Jaspers, eignen sich diese Praktiken jedoch kaum. Vielmehr müsse ein potenzielles Sein des Patienten durch eine existenzielle Kommunikation - eine Schicksalsgemeinschaft aus therapierender und therapierter Person - erarbeitet werden. ${ }^{44}$ Dadurch entstehe eine neuartige Gesamtaufgabe der Psychotherapeut_innen, die sowohl spezifische soziale Situationen der Patient_innen als auch deren persönliches Schicksal und das sie umgebende Milieu in der Behandlung berücksichtigen müssten. Die Kommunikation zwischen Binswanger und Warburg kann aber auch als Vorläufer jener psychotherapeutischen Ausrichtung verstanden werden, die Binswanger 1942 als Daseinsanalyse bezeichnen wird. ${ }^{45}$ Stimilli zeigt diesbezüglich auf, wie mit einer späteren Schrift Binswangers von 1956 die Behandlung Warburgs als Vorversion der Daseinsanalyse verstanden werden kann, denn dieser unterbreite in Drei Formen mißglückten Daseins «eine Definition von Heilung, die paradoxerweise die Definition eines künstlerischen Stils paraphrasiert, nämlich des Manierismus, wie sie im Essay <Zur Physiognomik des Manierismus〉 von Wilhelm Pinder steht $\gg{ }^{\mathbf{4 6}}$ Diese Form der Heilung sei aus daseinsanalytischer Perspektive gelungen, wenn Warburg es vollbringe, die Krankheit zum Erkenntnisgegenstand seines Selbst zu machen:

[Die] Schicksalsfrage [besteht] darin, ob es, um mit Pinder zu sprechen, gelingt, die $<$ kranke Lebenssphäre〉 zum <Gegenstand〉 zu machen, m. a. W. die kranke Individualität darin zu bringen, daß sie die 〈kranke Lebenssphäre 〈durchschaut〉. In der Psychiatrie sprechen wir dann von Krankheitseinsicht, und wenn sie durchbricht, sehen wir den Kranken als <gerettet> an. ${ }^{47}$

Bevor bei Warburg allerdings von einer Rettung gesprochen werden kann, gilt es im Sinne des Milieuwechsels zunächst einen Abstand zwischen ihm und seinen Gewohnheiten, also zwischen seinem psychotischen Selbst und seinen Organisationstechniken aufzubauen.

Die am 25. April r92 I, zu Beginn der Zeit Warburgs in Kreuzlingen, vorgenommene Tageseinteilung, «um allmählich etwas Ordnung in seine Lebensweise zu bringen», ${ }^{48}$ beschränkt sich auf Mahlzeiten, Waschzeiten und «Ruhen im Schlafzimmer».99 Doch abgesehen von wenigen ruhigeren Tagen Warburgs in seinem ersten Kreuzlinger Sommer bleibt seine Verfassung weitgehend wahnhaft. Am 4. Juni I92 I notiert Binswanger: «Jede kleine Veränderung löst neue Ausbrüche aus. In der Annahme, ihm eine Freude zu machen, wurden ihm ein Stoß seiner wissenschaftlichen Bücher, die erst jetzt über die Grenze gelangten, ins Zimmer gebracht».50 Anstatt sich über die Bücher zu freuen, regt Warburg sich jedoch «maßlos auf, da kein Verzeichnis dabei sei, ein Motiv, mit dem er offenbar nur die Erregung über das Neue rationalisiert». ${ }^{51}$ Die Krankengeschichte Warburgs, die von Ludwig und Kurt Binswanger beschrieben wird, touchiert hier erstmals jene Tinctura Warburgii, welche eine «Heilung mit Defekt $\gg^{\mathbf{5}}$ Warburgs bewirkt: die wissenschaftliche Arbeit. 
Am 25. März 1922 ist Warburg «hocherfreut, daß zwei Arbeiten, die er seinerzeit in Rom schrieb, nun im Druck erschienen sind. Sehr stolz darauf. Das wissenschaftliche Interesse wird überhaupt immer mehr wach; ab und zu diktiert er unserer Sekretärin diesbezügliche Briefe an Dr. Saxl.»53 Doch scheint die Erstdiagnose Binswangers sich zunächst als selbsterfüllende Prophezeiung zu bestätigen, wonach Warburg zwar stabilisiert, jedoch nie vollständig geheilt werden könne. Die Erregungszustände und Wahnvorstellungen schwanken in ihrer Intensität, verschwinden aber keinesfalls durch die Rückkehr zur Wissenschaft. Im Mai 1922 ist Warburgs «Befinden sehr wechselnd. Man hat den Eindruck, daß Aufregung fast schlimmer geworden ist in der letzten Zeit, daß aber Patient sich doch schneller beruhigen kann als früher, auch an schlechten Tagen sich unvermittelt ablenken läßt, meistens auf ein wissenschaftliches Thema». ${ }^{54}$ Binswangers Zeilen müssen im Zusammenhang mit der Diagnose verstanden werden, welche wie Chantal Marazia anmerkt, in direkter Verbindung zur Prognose stehen:

Der Begriff der Heilung ist an sich schon zweideutig. Wie in den anderen Zweigen der Medizin, so ist auch in der Psychiatrie die Prognose untrennbar mit der Diagnose verbunden. Am Fall Warburg ließe sich die aufsteigende Kurve von Binswangers Optimismus nachzeichnen, indem man die beiden unterschiedlichen geäußerten Diagnosen als Variablen verwendete. Solange Binswanger an der Eigendiagnose festhält, kann er eine <Wiederherstellung des Zustandes quo ante der akuten Psychose> nur ausschließen. Erst die spätere Hypothese, es könnte sich um einen <manisch-depressiven Mischzustand> handeln, gewährleistet die Möglichkeit einer Heilung. ${ }^{55}$

Das Behandlungsstadium einer Existenz-entwerfenden Kommunikation wird bei Warburg im Laufe der Kreuzlinger Jahre schrittweise etabliert und umfasst neben Gesprächen mit dem Therapeuten Binswanger, die via Brief noch lange nach Warburgs Entlassung weitergeführt werden, ganz substanziell auch die Rückgewinnung der eigenen Organisationstechniken in Verbindung mit kleineren wissenschaftlichen Ausarbeitungen und Vortragsentwürfen. Das Pharmakon ist bei Warburg die Schrift (wie bei Derrida) und zur Zeit des Briefes an seinen Bruder Paul zum Heilmittel dosiert. Die Genesung durch das dosierte Pharmakon wird ab jenem Zeitpunkt denkbar, als Warburg durch Anpassung der Diagnose eine Chance der Rekonvaleszenz zugesprochen wird und sich damit eine potenzielle Existenz realistisch entwerfen lässt. Nach der Untersuchung Warburgs durch den Psychiater Emil Kraepelin im Februar I923 steht Binswangers Befund der Schizophrenie im Krankenblatt nur noch in Klammern und wird durch Kraepelins Diagnose eines manischdepressiven Mischzustandes substituiert. ${ }^{56}$ Obwohl der angepasste Befund wesentlich bessere Prognosen für den Geisteszustand Warburgs impliziert, ${ }^{57}$ zeigt die anschließende Opiumkur keine Wirkung: «I8. März I923 - Schluß der Opiumkur. Dieselbe hat keine Beruhigung herbeigeführt. Patient war während derselben so schlecht wie noch nie».58 Saxls Besuch, eine knappe
53 Binswanger, Warburg: Die unendliche Heilung, 59 .

54 Ebd., 6 o.

55 Chantal Marazia: Heil und Heilung, in: Binswanger, Warburg: Die unendliche Heilung, 279-284, hier 280 .

56 Vgl. ebd., 252

57 Vgl. ebd., 76.

58 Ebd., 78. Und auch DidiHuberman stellt heraus, wie die Notizen für Warburgs ersten Vortrag nach Kreuzlingen von den Erfahrungen der Opiumkur zeugen, die das Opiat als Pharmakon disqualifizieren: «Das erste Wort des Entwurfs lautet: ‘Hilfe!’. Es folgt ein pharmakologischer Hinweis: (... noch unter Opiumkur geschrieben'", Didi-Huberman: Das Nachleben der Bilder, 403. 
Woche nach der missglückten Behandlung, wird von Binswanger allerdings positiv bewertet: «Der Besuch Dr. Saxls verläuft befriedigend. Patient ist wieder viel ruhiger geworden. Er arbeitet ziemlich regelmäßig. Vormittags $1 / 2$ bis I Stunde, nachmittags 2 Stunden mit Dr. Saxl an seinem Vortrag, geht mit ihm gemeinsam spazieren».59 Der unterschiedliche Erfolg der Behandlungen mit Opium respektive mit wissenschaftlicher Tätigkeit verdeutlicht, welches Pharmakon bei Warburg produktiver wirkt. In dem zum Pharmazeutikum regulierten Ensemble von Organisations-, Wissens- und Formationstechniken des Privatgelehrten wird das Potenzial einer Medientheorie erkennbar, die im Zusammenwirken von Warburg und Derrida und zwischen Formation und Pharmakon entsteht:

Es gibt kein harmloses Heilmittel. Das pharmakon kann niemals einfach wohltuend sein. [...] Zuallererst, weil das wohltuende Wesen oder die wohltuende Kraft des pharmakon es nicht daran hindert, schmerzhaft zu sein. [...] Dieser mit der Krankheit wie auch mit ihrer Linderung verbundene schmerzhafte Genuß ist bereits an sich ein pharmakon. Er partizipiert gleichzeitig am Guten und am Bösen, am Angenehmen und am Unangenehmen. Oder eher noch: in seiner Massierung zeichnen sich diese Gegensätze ab. ${ }^{\mathbf{6 0}}$

Mario Wimmer hat den von Warburg geschaffenen Begriff der «Gehirnausstülpung» als Motiv für eine Wissenschaftstheorie produktiv gemacht. ${ }^{61} \mathrm{Nach}$ Wimmer lässt sich an diesem Begriff aufzeigen, wie sehr sich intellektuelle Arbeit durch ein Zusammenspiel von Entwurf und Affekt vollzieht. Ganz im Sinne eines Experimentalsystems bleibe es immer fraglich, ob ein wissenschaftlicher Prozess zu validen Ergebnissen führe: «Erst so hebt sich der Zwang, Kopf zu sein, auf und ermöglicht ein Denken, das mit dem operiert, was zuhanden ist und zugleich jenen notwendigen Affekt hervorbringt, der durch vorübergehend stabile Arrangements erst entsteht» ${ }^{62}$ Die Substanz des Pharmakons ist nach Derrida jedoch zumeist flüssig, wie «[d]as Sperma, das Wasser, die Tinte, die Farbe, der parfümierte Anstrich: das pharmakon dringt stets als das Flüssige ein, läßt sich trinken, aufsaugen, ins Innere einführen und markiert dieses zunächst mit der Festigkeit des Typos, um es alsdann mit seiner Arznei, seinem Arzneitrank, seinem Getränk, seiner Mixtur, seinem Gift zu überschwemmen und zu überfluten». ${ }^{63} \mathrm{Im}$ Fall Warburgs sind es wirkmächtige Ordnungstechniken des Wissens, die Warburgs Psyche zeitweise ordnen und arrangieren, jedoch auch die Gefahr bergen, den Gelehr-

59 Binswanger, Warburg: Die unendliche Heilung, 78.

60 Derrida: Dissemination, 111. 61 Mario Wimmer: Gehirnausstülpungen. Zur Wissenschaftsgeschichte intellektueller Arbeit, in: Berichte zur Wissenschaftsgeschichte, Bd. 41, Nr. 4, 2018, 449-452, hier 449 . 62 Ebd., 451.

63 Derrida: Dissemination, 170. ten mit ihrer Macht zu überfluten. Durch das Format-Schaffen entsteht ein Pharmakon, welches Denken und Wissenschaft in eine fluide Formation des Un/Ordentlichen transformiert. Im Gegensatz zum Pharmakon-Konzept Derridas, das explizit im Zustand des Flüssigen gedacht wird, bildet Warburgs Pharmakon aber nachweislich auch Materielles (Bildatlas, Zettelkasten, Bibliothekstagebuch), wie es auch seine Materien mitbildet. Eine Medientheorie des Formate-Schaffens könnte das Pharmakon vielversprechend in Stellung 
bringen, für eine Theorie von Schreibprozessen als Existenzformationen, die fluide Formationen und handfeste Formate des Daseins vereint:

Wissenschaft und Magie, Übergang zwischen Leben und Tod, Supplement des Übels und des Mangels: die Medizin sollte Thoths bevorzugte Domäne bilden. Alle seine Mächte kamen darin zusammen und zur Anwendung. Der Gott der Schrift, der dem Leben ein Ende zu setzen weiß, heilt auch die Kranken. Und sogar die Toten. [...] Der Gott der Schrift ist also ein Gott der Medizin. ${ }^{64}$ 